\title{
PROYECTO RALET DE LA RED DE LUDOTECAS MUNICIPALES DEL AYUNTAMIENTO DE LLEIDA
}

\author{
Teresa Bitria Figuera \\ Ayuntamiento de Lleida \\ tbitria@paeria.es \\ Ester Pascual Arderiu \\ Ayuntamiento de Lleida \\ epascual@paeria.es
}

Fecha de Recepción: 18 Abril 2019

Fecha de Admisión: 30 Abril 2019

\section{RESUMEN}

Nuestro Proyecto se enmarca dentro del Programa LIF (Ludoteca, infancia y familia). El Proyecto Ralet, es uno de los proyectos que se ofrecen des de la Red de Ludotecas Municipales dirigidos a Primera Infancia.

Nuestros objetivos son siguientes:

- Construir una red de apoyo entre las familias participantes.

- Conocer el desarrollo del niño y sus funciones principales en la primera infancia.

- Conocer herramientas educativas para la intervención en el desarrollo de sus hijos /as de acuerdo a la edad del niño/a.

- Dar a conocer diferentes recursos que existen en la ciudad de Lleida.

- Observar la dinámica familiar y el desarrollo del niño/a para poder prevenir y detectar situaciones de riesgo.

Este proyecto fue evaluado con el "Protocolo de buenas prácticas en el enfoque de la parentalidad positiva" y la recomendación para el servicio que elegimos fue:

BP4 Seguir una estrategia preventiva y no sólo reparadora en el servicio.

El servició debería destinar tiempo para detectar las necesidades y fortalezas de las familias en la comunidad con el fin de preparar planes de actuación preventivos en el ámbito comunitario.

En base a esta necesidad diseñamos un plan de mejora basado en destinar un espacio para detectar necesidades y fortalezas. El proyecto que consta de diez sesiones contempla un espacio físico y temporal donde compartir sus preocupaciones, intereses, motivaciones etc.

Para poder realizar esta estrategia se movilizaron una serie de recursos personales, materiales, económicos y de formación y cuando acabamos se evaluó el progreso de la prioridad seleccionada.

Palabras clave: primera infancia; red de apoyo; familias; desarrollo del niño/a; estrategia preventiva 


\section{ABSTRACT}

The ralet project of the municipal playrooms network of the Lleida city council. Our project is part of the LIF program (playroom, childhood and family). The Ralet project is one of the projects offered by the network of municipal play centres aimed at early childhood.

Our objectives are as follows:

- Build a support network among participating families.

- Know child development and its main functions in early childhood.

.- Know educational tools for intervention in the development of their children according to the age of the child.

-Know different resources that exist in the city of Lleida.

-Observe family dynamics and child development in order to prevent and detect risk situations.

This project was evaluated with the "Protocol of good practices in the approach of positive parenting" and the recommendation for the service we chose was the following:

BP4. To follow a preventive strategy and not only restorative in the service.

The programme should be used to identify the needs and strengths of families in the community in order to prepare preventive action plans at the community level.

Based on this need, we designed an improvement plan based on allocating space to detect needs and strengths. The project that consists of ten sessions contemplates a physical and temporal space where to share their worries, interests, motivations etc. In order to be able to carry out this strategy, a series of personal, material, economic and training resources were mobilised and, when we finished, the progress of the selected priority was evaluated.

Keywords: early childhood; support network; families; development of their children; preventive strategy

\section{"PROTOCOLO DE BUENAS PRÁCTICAS EN PARENTALIDAD POSITIVA". EXPERIENCIA DE APLICACIÓN EN EL PROYECTO RALE}

El Ayuntamiento de Lleida y la Concejalía de Educación llevan años impulsando el desarrollo de programas donde las familias son las principales protagonistas, ya que des de hace un tiempo que se considera a la familia la primera institución socializadora.

Nuestro Proyecto se enmarca dentro del Programa LIF (Ludoteca, infancia y familia). El Proyecto Ralet, es uno de los proyectos que se ofrecen des de la Red de Ludotecas Municipales dirigidos a Primera Infancia. El programa LIF también incluye otros proyectos aparte del nuestro: Proyecto Nadó, cuya esencia ve continuidad en los que les siguen como, Proyecto Ralet, Proyecto FIL (Familia y Ludoteca) y Ludoteca Familiar, donde acompañamiento y juego son los ejes principales.

Estos proyectos pueden iniciarse des del nacimiento. El primer proyecto al cual se puede acceder es el Proyecto Nadó (de 0 a 6 meses) donde les familias pueden compartir sus primeras experiencias y lo que se pretende des del proyecto es acogerlas y crear unos vínculos que favorezcan una red de apoyo entre las familias participantes.

El siguiente proyecto a partir de los seis meses es el proyecto Ralet, que es en el que se aplicó el Protocolo de Buenas Prácticas en Parentalidad Positiva y sobre el que nosotros profundizaremos más adelante.

A partir del año y hasta los tres años se ofrece el Proyecto FII, donde el niño pasa a ser el protagonista de la intervención educativa sin olvidar la implicación de la familia.

Des de la primera edición del Proyecto en 2008, los contenidos de las sesiones se han ido modificando en función de los cambios en los modelos de crianza así como las evaluaciones que las familias realizan al final de cada edición. 
Los objectivos del Proyecto Ralet son los siguientes:

- Acoger a las familias que acaban de tener un hijo/a.

- Construir una red de apoyo entre las familias participantes.

- Conocer el desarrollo del niño y sus funciones principales en la primera infancia.

- Conocer herramientas educativas para la intervención en el desarrollo de sus hijos /as de acuerdo a la edad del niño/a.

- Dar a conocer diferentes recursos que existen en la ciudad de Lleida.

-- Observar la dinámica familiar y el desarrollo del niño/a para poder prevenir y detectar situaciones de riesgo.

La tipología de familias que se inscriben en nuestros proyectos provienen de un entorno sociocultural y socioeconómico medio, en principio no son familias en riesgo de exclusión social.

La metodología del proyecto es participativa y activa para que las familias se sientan agentes implicados en el proyecto de forma integral.

Tan o más importante que el contenido de las sesiones y la información que se comparte, es el clima que se establece, así como la oportunidad de participación que se ofrece a las familias para que puedan expresar sus inquietudes, emociones, opiniones, conocimientos sobre los diferentes temas propuestos, todo a través de una mirada respetuosa, libre de juicios, des de una escucha activa que dé pie a todos los miembros del grupo a participar si así lo desean.

Como hemos dicho en los objetivos anteriores, la clave de éxito de este proyecto es la doble vertiente, por un lado, ofrecer en este espacio libre de juicio en que poder crear tribu y desarrollar cierto sentimiento de pertinencia, y por otro lado, dotar a los padres y madres de ideas y recursos para capacitarlos de manera que dispongan de cierta información, pero además sepan donde ampliarla.

Estos espacios surgen de la necesidad de ofrecer a las nuevas madres y padres una red de apoyo. En la sociedad actual la maternidad se vive a menudo de forma solitaria como consecuencia de un modelo familiar en que la familia extensa ha desaparecido, así como la red de mujeres entorno a la maternidad que apoyaba a la futura madre en embarazo, parto y puerperio, y que venía siendo habitual en la historia de la humanidad.

Por otra parte es bien sabido que la información empodera a quien la recibe de manera que compartiendo los recursos y concomimientos de esta etapa de sus bebés, infundimos seguridad y dotamos de confianza a padres y madres para que puedan afrontar de sus hijos.

En nuestra búsqueda constante de mejorar nuestra intervención socio-educativa se nos propuso en el 2017 participar en las "Jornadas de formación de profesionales en la Guía de Buenas Prácticas en Parentalidad Positiva y el uso del protocolo online" en Valladolid.

A través de esta formación en Parentalidad Positiva descubrimos que este enfoque legitima y sustenta nuestra intervención socio-educativa, ya que los principios en los que se basa se identifican con nuestro proyecto y nos dan herramientas para promover aspectos de la Parentalidad Positiva que conforman la columna vertebral de nuestra actividad diaria.

A raíz de esta formación, creamos un grupo de trabajo formado por tres técnicas de Educación responsables del Proyecto Ralet y implementamos el Protocolo para evaluar y analizar nuestro proyecto según la aplicación del enfoque de la Parentalidad Positiva.

El grupo dinamizador procedió a responder de manera ordenada el Protocolo y estos fueron los resultados en los diferentes bloques:

\section{PARTE 1. Buenas prácticas en el servicio des del enfoque de la parentalidad positiva.}

BP1: Plantear los objetivos del servicio des de una perspectiva de derechos y necesidades de desarrollo des de la infancia y adolescencia. (3.71). 
BP2: Favorecer la promoción del ejercicio de la parentalidad positiva en el servició (3.5).

BP3: Establecer canales de sensibilización e información para facilitar el acceso universal al servició (2.67).

BP4: Seguir una estrategia preventiva y no sólo reparadora en el servicio (2.82).

BP5: Emplear modelos y prácticas profesionales basadas en el consenso y la evidencia científica (3.43).

BP6: Disponer de una estructura organizativa y unas condiciones de trabajo que permitan el apoyo a la parentalidad positiva (3.18).

BP7: Identificar las competencias profesionales necesarias para el trabajo con familias (3.78).

BP8: Promover el trabajo con otras instituciones que también intervienen con infancia, adolescencia y familia (2.67).

BP9: Potenciar la colaboración con el ámbito universitario, tanto para el asesoramiento como para la investigación en parentalidad positiva. (3.2)

Las recomendaciones para el servicio des de este enfoque fueron para:

BP3: Establecer canales de sensibilización e información para facilitar el acceso universal al servició (2.67).

BP4: Seguir una estrategia preventiva y no sólo reparadora en el servicio (2.82).

El servicio debería destinar tiempo para detectar las necesidades y fortalezas de las familias en la comunidad con el fin de preparar planes de actuación preventivos en el ámbito comunitario.

La recomendación que hemos tenido en cuenta y posteriormente ampliaremos es esta última.

\section{PARTE 2. Buenas prácticas en el trabajo profesional con las familias des del enfoque de la parentalidad positiva.}

BP10: Establecer una relación con las familias basada en la confianza y el respeto mutuo. (2.08).

BP11: Analizar/ evaluar las necesidades de apoyo y fortalezas a nivel personal y familiar. (2.35).

BP12: Analizar/evaluar las competencias parentales implicadas en el desempeño de la parentalidad positiva. (2.75).

BP13: Analizar/avaluar las necesidades de desarrollo y las competencias de los niños, niñas y adolescentes. (3.57).

BP14: Llevar a cabo una evaluación rigurosa de las condiciones en las que se ejerce la parentalidad en las familias. (3.00).

BP15: Mantener una comunicación con las familias que potencie el reconocimiento de sus fortalezas durante la intervención. (3.17).

BP16: Buscar la colaboración de toda la familia para realizar una intervención realista y consensuada con las familias. (3.86).

BP17: Realizar actividades estructuradas en la atención individual con las familias. (3.05).

BP18: Realizar actividades grupales estructuradas para la promoción de la parentalidad. (3.67).

BP19: Realizar actividades grupales estructuradas para la atención de la infancia y la adolescencia. (3.43).

BP20: Realizar actividades estructuradas para la atención comunitaria. (3.29).

Las recomendaciones para el servicio des de este enfoque fueron para:

BP10: Establecer una relación con las familias basada en la confianza y el respeto mutuo. (2.08).

BP12: Analizar/evaluar las competencias parentales implicadas en el desempeño de la parentalidad positiva. (2.75).

BP15: Mantener una comunicación con las familias que potencie el reconocimiento de sus fortalezas durante la intervención. (3.17). 
PARTE 3. Buenas prácticas en los programas basados en evidencias des del enfoque de la parentalidad positiva.

BP21: Fundamentar el programa en una base científica y formular objetivos claros y susceptibles de ser medidos. (3.6).

BP22: Desarrollar el programa con una metodología bien diseñada para la atención individual, grupal o comunitaria. (3.5).

BP23: Coordinar y dinamizar el grupo de forma eficaz fomentando un clima de relación positivo en el programa grupal. (3.52).

BP24: Evaluar el programa con criterios científicos. (4).

BP25: Incorporar el programa a la comunidad y contribuir a su desarrollo. (3.67).

En esta tercera parte las recomendaciones que obtuvimos para el Programa Proyecto Ralet fueron:

BP24: Evaluar el programa con criterios científicos. (4).

BP25: Incorporar el programa a la comunidad y contribuir a su desarrollo. (3.67).

\section{PLAN DE MEJORA PARA EL PROYECTO RALET DE LA RED DE LUDOTECAS MUNICIPALES DE LLEIDA.}

\section{PRIORIDAD ELEGIDA}

Nosotras hemos elegido como prioridad seguir una estrategia preventiva y no sólo reparadora en el servicio. Tenemos muy presente, que el ejercicio de la parentalidad positiva no se ejerce en un espacio vacío sino que requiere de aliados y de apoyos materiales, de información y consejo, de acompañamiento, de comprensión y de carácter formativo para mejorar su ejercicio. Todos estos apoyos, que proporcionan personas e instituciones, favorecen el bienestar físico y psicológico de los miembros de la familia, reduciendo el impacto de los acontecimientos estresantes y promoviendo un sentimiento de identidad de autoestima y de integración a la sociedad. Ya que es necesario identificar las necesidades de apoyo que tienen las familias para ejercer la parentalidad y trabajar con ellas y con los hijos e hijos para reforzar sus capacidades respectivas con el fin de alcanzar las metas de desarrollo adecuadas. ${ }^{1}$

Por estos motivos creemos que nuestro proyecto debería destinar tiempo para detectar las necesidades y fortalezas de las familias en la comunidad con el fin de preparar planes de actuación preventivos en el ámbito comunitario.

Nuestro proyecto pretende acoger a familias con bebes de 6 hasta 12 meses para ofrecer un espacio de interrelación en esta etapa vital, el nacimiento de un hijo o hija es sin duda una de las experiencias más maravillosas en la vida de una persona. La llegada de un bebé abre una nueva etapa llena de ilusiones pero también de incertidumbre y por qué no decirlo de inquietudes que suponen los nuevos retos.

La familia primer referente del niño, juega un papel insustituible en la formación de su identidad y en la relación con otros niños. Es el espacio principal para la crianza de los menores en una etapa primordial, de 0 a 36 meses, cuando se establece el vínculo afectivo y se construye la identidad del niño. (VVAA: 2007) ${ }^{2}$

\section{ACCIÓN A REALIZAR}

El proyecto consta de 10 sesiones semanales de hora y media de duración durante las cuales se proponen temas de crianza y del momento en que se encuentran las familias asistentes.

El grupo consta de 12 a 15 familias ya que consideramos que es un número suficiente para ofrecer disparidad y diversidad pero no lo suficiente para que se pierda calidez, confortabilidad la 
esencia del proyecto. Los temas propuestos comprenden el desarrollo de los sentidos y los primeros juegos, música, alimentación complementaria, parentalidad positiva.... focalizado en como el adulto acompaña al bebé en las diferentes áreas. Las sesiones son desarrolladas por las educadoras al cargo en su mayoría, o bien, por profesionales externos que colaboran en él.

Los tiempos han cambiado y también el concepto de convivencia y educación en familia. El contexto social y económico ha hecho que las unidades familiares hayan cambiado en estructura, en demandas, necesidades y también en la dedicación a la crianza del bebe y en su bienestar educativo y emocional.

Así, la experiencia maravillosa de la llegada de una criatura a la familia y el cumulo de sentimientos y sensaciones positivas que comporta también se convierten en un desafío, no exento de momentos de confusión y dificultad, son muchas las dudas y la necesidad de información que genera esta compleja tarea que es la crianza. De aquí que nuestra propuesta de acción sea que en cada sesión se destine un espacio a detectar las necesidades que se derivan de esta etapa vital: inquietudes, emociones negativas, estrés, sentimientos de pérdida, estado físico, el post parto, los sentimientos ambivalentes, ...

Al iniciar cada sesión se pregunta a las familias si tienen alguna inquietud que quieran expresar de forma libre relacionada con la crianza, así como alguna duda no resuelta de sesiones anteriores. Para poder facilitar la dinámica comunicativa se ofrece la posibilidad de anotar sugerencias o preocupaciones que en próximas sesiones se pueden abordar.

Estos factores que en un principio no tienen por qué ser de riesgo pueden derivar en algunas familias en estresores psicosociales que complican la tarea de ser padres.

Nuestra acción iría dirigida a la promoción de las competencias parentales, dotar a los padres y madres de ideas y recursos para capacitarlos de manera que puedan cubrir las necesidades físicas, psicológicas y sociales de sus hijos aprovechando las oportunidades y apoyos sociales de su entorno.

Por otra parte, es bien sabido que la información empodera a quien la recibe de manera que compartiendo los recursos y concomimientos de esta etapa de sus bebe, infundimos seguridad y dotamos de confianza a padres y madres para que puedan afrontar la crianza de sus hijos.

\section{TIEMPO DE CONSECUCIÓN}

Fecha de inicio: 2 de abril de 2018.

Fecha de finalización: 20 de junio del 2018.

\section{RECURSOS A EMPLEAR}

Recursos personales: Somos un equipo formado por tres educadoras que iniciamos el Proyecto Ralet en el 2008 de manera continuada hasta el día de hoy, nuestra formación continua nos ha permitido adaptarnos a los diferentes retos que plantea los modelos de crianza actuales. En el 2017 se nos planteó una formación en parentalidad positiva que nos hizo reflexionar sobre como introducir aspectos de este enfoque en nuestro proyecto, ya que los principios en los que se basa van en la línea de nuestro trabajo. (Rodrigo y Palacios, 1998). ${ }^{3}$

Recursos materiales: Crear un espacio acogedor donde se establece un clima de seguridad y protección, decorado teniendo en cuenta las necesidades psicopedagógicas de las familias y con una elección de materiales rigurosa y con criterio pedagógico.

Recursos económicos: Presupuesto que se nos destina anualmente a nuestro servicio. De forma puntual si se requiere el Proyecto ha recibido partidas presupuestarias específicas para necesidades concretas. 


\section{NECESIDADES DE FORMACIÓN DETECTADAS}

FORMACIÓN PRESENCIAL DIRIGIDA A LOS PROFESIONALES CON DOCENTE EXTERNO.

Después de aplicar el Protocolo de Buenas Prácticas y teniendo en cuenta la necesidad que íbamos a trabajar decidimos pedir formaciones relacionadas con el papel del profesional en temas de escucha activa, crianza respetuosa basada en la Metodología Pickler y Disciplina Positiva en primera infancia.

\section{FORMACIÓN ONLINE DIRIGIDA A LOS PROFESIONALES.}

En formación online complementamos la formación en parentalidad positiva realizando el curso online "Ganar Salud y bienestar de 0 a 3 años". Después de realizar este curso y ver sus beneficios lo hemos introducido en la documentación que damos a las familias al finalizar nuestro proyecto cómo herramienta de información y formación. Con el fin de adquirir recursos de formación y capacitación dirigidos a fomentar modelos parentales positivos para que niños y niñas puedan desarrollar su máximo potencial.

\section{MODO DE EVALUACIÓN DEL PROGRESO DE LA PRIORIDAD SELECCIONADA:}

Los indicadores en los que nos fijaremos para valorar el logro de la prioridad al finalizar el proyecto son:

- Grado de manifestación de las necesidades por parte de las familias.

- Grado de manifestación de los logros por parte de las familias.

- Grado de satisfacción de las familias respecto a la respuesta a sus necesidades.

- Grado de satisfacción de las familias respecto a la valoración de sus fortalezas.

Con el fin de evaluar los indicadores mencionados al inicio del proyecto se pasará un cuestionario a las familias para detectar las necesidades y fortalezas y al finalizar se pasará otro.

En el ecuador del proyecto realizaremos un "brainstorming" con las familias para compartir entre ellas la evolución de las necesidades y fortalezas a lo largo del curso.

Al finalizar el proyecto se hará un retorno a las familias donde se indicaran los recursos personales (internos y externos) con los que puede contar cada familia y así seguiremos una estrategia preventiva, ya que en una primera fase el objetivo será identificar las necesidades para ya después prevenir situaciones posibles futuras.

\section{CONCLUSIONES}

Las familias participantes expresaron un alto grado de satisfacción respecto al Proyecto, y valoraron muy positivamente el hecho de poder dispones de un instrumento de apoyo y posibilidad de expresión libre de juicios donde poder manifestar sus opiniones y inquietudes. Esta práctica ya la llevábamos a cabo pero al finalizar el proyecto y de manera puntual en alguna sesión y de manera verbal, esta doble fórmula escrita y verbal nos permite conocer la opinión y las preocupaciones de la mayoría de las familias. Al crearse estas condiciones hemos podido profundizar de forma más real y exacta en las diferentes problemáticas que atraviesan las familias en esta etapa.

Para concluir este proceso decir que nos encontramos en una fase de reflexión hacia el enfoque de la parentalidad positiva de forma glogal, teniendo en cuenta la posibilidad de expandir el enfoque a I resto de proyectos que se hacen en nuestro programa LIF.

\section{REFERENCIAS BIBLIOGRÁFICAS}

VVAA. (2007). Crèixer amb tu. Els infants de 0 a 18 mesos. Barcelona: Generalitat de Catalunya. WEBGRAFIA

http://familiasenpositivo.org/parentalidad-positiva 
1 http://familiasenpositivo.org/parentalidad-positiva/necesidades

2 VVAA. (2007). Crèixer amb tu. Els infants de 0 a 18 mesos. Barcelona: Generalitat de Catalunya.

3 http://familiasenpositivo.org/parentalidad-positiva/principios 